\title{
Pure small-cell carcinoma of the prostate presenting with increasing prostate-specific antigen levels: A case report and review of the literature
}

\author{
JIA HU $^{1,2}$, TAO HE$^{1,2}$, LU JIN $^{1}$, YIFAN LI $^{1}$, YANG ZHAO $^{1}$, WENHUA LI ${ }^{1}$, BENLIN WEI $^{1}$, \\ XIANG MING MAO ${ }^{1}$, YONG QING LAI ${ }^{1 *}$ and LIANG CHAO NI ${ }^{1 *}$ \\ ${ }^{1}$ Department of Urology, Peking University Shenzhen Hospital, Shenzhen, Guangdong 518036; ${ }^{2}$ Department \\ of Urology, Guangzhou Medical University, Guangzhou, Guangdong 510182, P.R. China
}

Received August 11, 2017; Accepted May 16, 2018

DOI: $10.3892 / \mathrm{mco} .2018 .1644$

\begin{abstract}
The incidence of prostatic cancer ( $\mathrm{PCa}$ ) has increased significantly, and the measurement of prostate-specific antigen (PSA) is an effective screening tool for its diagnosis. PCa includes a number of specific clinicopathological types, including squamous cell, urothelial, adenoid cystic and small-cell carcinoma, among which small-cell carcinoma of the prostate (SCCP) is extremely rare, accounting for $<0.5 \%$ of all PCa cases. SCCP is very aggressive and the majority of the cases have a poor prognosis, with a mean survival of $\sim 5$ months; it also exhibits specific clinicopathological characteristics and may be divided into two subtypes, namely pure and mixed SCCP. According to the previous literature on PubMed, pure SCCP is not associated with an increase in serum PSA levels. However, the rare case presented herein exhibited an increasingly abnormal serum PSA level. The patient was aged 66 years and had a PSA level of $56.78 \mathrm{ng} / \mathrm{ml}$ (normal, <4 ng/ml); he was diagnosed with pure SCCP, underwent radical prostatectomy and has remained disease-free during the follow-up. Similar cases previously published on PubMed were also reviewed, and considerations of survival status and treatment options were analyzed.
\end{abstract}

\section{Introduction}

The first case of SCCP was reported in 1977 (1). It has since been reported that a number of patients have a history of a

Correspondence to: Professor Liangchao Ni or Professor Yong Qing Lai, Department of Urology, Peking University Shenzhen Hospital, 1120 Lianhua Road, Shenzhen, Guangdong 518036, P.R. China

E-mail: 13609618222@163.com

E-mail: yqlord@163.com

*Contributed equally

Key words: prostatic carcinoma, small-cell carcinoma, pure, prognosis hormonally treated acinar adenocarcinoma. SCCP is histologically similar to small-cell carcinoma of the lung (SCCL) (2). In terms of distinguishing between SCCP and a metastatic tumor from the lung, however, the results are conflicting as to whether SCCP is positive for thyroid transcription factor-1 (TTF-1). As the pure SCCP component predominates, as reported among different studies, the serum prostate-specific antigen (PSA) level may be undetectable. There is no difference in prognosis between patients with pure and mixed (with an acinar adenocarcinoma component) SCCP (1). Surgery and clinical staging are not correlated with prognosis. We herein present a rare case of a patient who was diagnosed with pure SCCP, with a serum PSA level of $56.78 \mathrm{ng} / \mathrm{ml}$. To the best of our knowledge, among the 21 cases of pure SCCP published on PubMed, our patient had the highest serum PSA level reported to date for this type of tumor $(1,3)$.

\section{Case report}

A 66-year-old man was admitted to the Department of Urology of Peking University Shenzhen Hospital in March 2015 due to elevated serum PSA level.

The transrectal ultrasound examination revealed an irregularly enlarged prostate. The findings on chest X-ray and laboratory biochemical tests, including routine complete blood count, serum biochemical analysis and urinalysis, were unremarkable, except for an abnormally elevated PSA value $(56.78 \mathrm{ng} / \mathrm{ml}$; normal, $<4 \mathrm{ng} / \mathrm{ml})$. The patient subsequently underwent transrectal ultrasound-guided prostate biopsy, which revealed a Gleason score of $3+4=7$. An abdominal and pelvic computed tomography examination was performed, which revealed a prostatic mass, without signs of distant metastasis (Fig. 1A). The preoperative stage in our case was T2N0M0. The patient was diagnosed with a preinvasive $\mathrm{PCa}$ and radical prostatectomy surgery was immediately initiated. Radical prostatectomy with regional lymph node dissection was performed straight after surgery. Macroscopic cancer invasion of periprostatic tissue was not detected intraoperatively.

The patient was discharged on postoperative day 7 without major complications (although minor complications included 

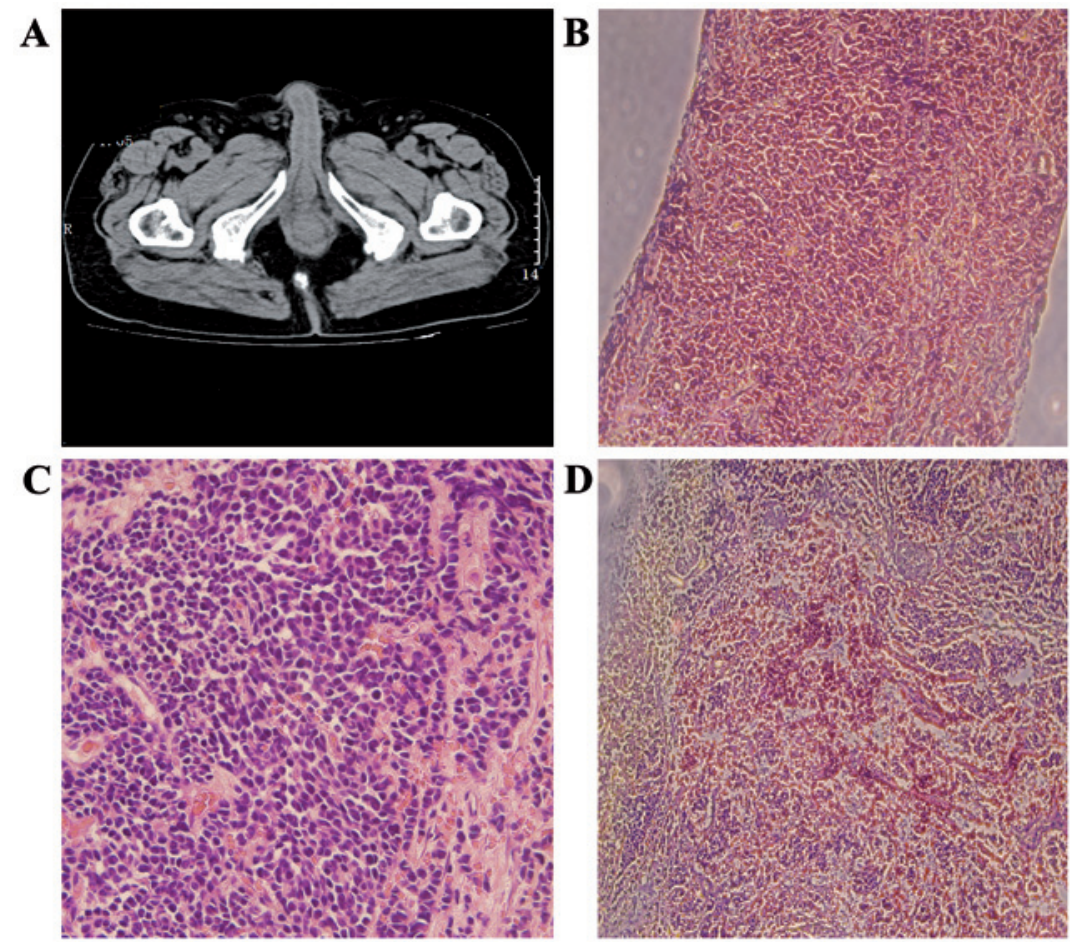

Figure 1. (A) A non-contrast-enhanced computed tomography scan of the pelvis revealed an uneven lump (20 HU) on the right lobe of the prostate. (B and C) On microscopic examination, the carcinoma cells were round or short spindle-shaped, arranged in a flaky or nest-like pattern. The cells contained scant cytoplasm, with nuclei lacking a covering of mitochondria or endoplasmie reticulum and deeply stained. Magnification, (B) x100 and (C) x400. (D) On immunohistochemical examination, the cells stained positive for thyroid transcription factor-1 (magnification, x100).

bleeding, pain and sleeplessness). The microscopic examination of the surgical specimen revealed the presence of pure (without foci of adenocarcinoma) SCCP (Fig. 1B and C). The immunohistochemical examination further confirmed the diagnosis, with a positive expression of TTF-1 (Fig. 1D). After the recommended postoperative treatment options were explained in detail, the patient refused further chemotherapy, radiotherapy and endocrine therapy. The serum PSA level was measured on a 3-month basis, while abdominal and pelvic contrast-enhanced CT was performed at 6 and 12 months postoperatively. On the multiple follow-up visits (the last in March 2016), the patient exhibited no signs of disease recurrence.

The publication of this case was approved by the Ethics Committee of Peking University Shenzhen Hospital, and written informed consent was obtained from the patient.

\section{Discussion}

SCCP is a rare entity, accounting for $<0.5 \%$ of all $\mathrm{PCa}$ cases (4). SCCP has an aggressive behavior and it tends to metastasize early to distant organs, such as the liver, bones, skin, bladder, rectum, lymph nodes, and even the lungs (4). SCCP usually arises from the peripheral zone of the prostate and may occur without obstructive symptoms of the urinary tract. SCCP may be divided into two subtypes (pure and mixed SCCP), according to the presence of an adenocarcinomatous element (1). Pure SCCP is an uncommon pathological pattern, with only few cases reported in the literature to date. The diagnosis is easily missed due to the normal PSA level (5). The main finding in our patient was the abnormal PSA level, which was inconsistent with previous studies.
The clinicopathological characteristics of pure SCCP are similar to those of SCCL (Table I). As in SCCL, vascular invasion, high-grade malignancy, high mitotic index and necrosis are common characteristics (6). Two possibilities regarding the histogenesis of SCCP were recently proposed (7). The most persuasive hypothesis is that pure SCCP is derived from totipotential stem cells, which may easily differentiate into neuroendocrine and epithelial types (7). Another theory is that small-cell cancer may arise from the amine precursor uptake or decarboxylation cells of the endoderm. The latter depends on the hypothesis that SCCP is part of the huge spectrum of the prostatic adenocarcinomas (7). Primary pure SCCP and metastatic carcinoma may be distinguished by the expression of TTF-1 (8). The confirmation of pure SCCP mainly relies on pathological examination. Microscopically, SCCP cells are round or short spindle-shaped, arranged in a flaky and nest-like pattern. The carcinoma cells contain scat cytoplasm, with nuclei situated far from mitochondria or endoplasmic reticulum (8). The nucleolus is blurry and mitotic figures may be observed; necrosis is also a common accompanying finding. Positive expression of synaptophysin, chromogranin A and CD56 on immunohistochemical examination is valuable for the diagnosis (9). The aforementioned criteria may be useful in differentiating pure SCCP from its mixed counterpart (9).

As the endocrine component of pure SCCP is similar to SCCL, paraneoplastic syndromes may be observed in a proportion of the patients, such as thyrotoxicosis, syndrome of inappropriate antidiuretic hormone secretion, hypercalcemia and Cushing's syndrome. The present case did not exhibit any symptoms or signs of paraneoplastic syndromes (10). The 
Table I. Clinicopathological characteristics of 22 cases of pure SCCP.

\begin{tabular}{|c|c|c|c|c|c|c|}
\hline Case no. & Age, years & PSA & Diagnostic procedure & Treatment & Follow-up, months & Survival status \\
\hline 1 & 76 & 0.81 & TURP & None & 6 & Deceased \\
\hline 2 & 32 & 0.45 & Radical & Chemotherapy & 10 & Deceased \\
\hline 3 & 61 & 2.4 & Biopsy & None & 1 & Lost \\
\hline 4 & 43 & 8.8 & Biopsy & Chemotherapy & 1 & Alive \\
\hline 5 & 56 & $<4$ & Radical & Endocrine therapy & 17 & Alive \\
\hline 6 & 32 & $<4$ & Biopsy & Chemotherapy & 3 & Lost \\
\hline 7 & 65 & $<4$ & Biopsy & Chemoradiotherapy & 11 & Deceased \\
\hline 8 & 21 & 0.69 & Biopsy & Endocrine therapy & 3 & Deceased \\
\hline 9 & 82 & 2.26 & TURP & Endocrine therapy & 3 & Deceased \\
\hline 10 & 61 & 0.32 & Radical & Chemotherapy & 3 & Alive \\
\hline 11 & 55 & $<4$ & Radical & Endocrine therapy & 3 & Deceased \\
\hline 12 & 34 & $<4$ & Biopsy & Chemotherapy & 13 & Deceased \\
\hline 13 & 62 & $<4$ & Radical & None & 5 & Alive \\
\hline 14 & 65 & 1.92 & Biopsy & $\begin{array}{c}\text { Chemotherapy + endocrine } \\
\text { therapy +radiotherapy }\end{array}$ & 11 & Deceased \\
\hline 15 & 55 & $<4$ & Radical & Endocrine therapy & 3 & Deceased \\
\hline 16 & 34 & $<4$ & Biopsy & Chemotherapy & 12 & Alive \\
\hline 17 & 50 & 0.31 & Radical & None & 1 & Deceased \\
\hline 18 & 82 & 2.61 & TURP & $\begin{array}{l}\text { Chemotherapy + endocrine } \\
\text { therapy +radiotherapy }\end{array}$ & 17 & Alive \\
\hline 19 & 81 & 39.26 & Biopsy & None & 5 & Deceased \\
\hline 20 & 77 & 25.02 & Biopsy & Chemoradiotherapy & 2 & Alive \\
\hline 21 & 76 & $<4$ & Biopsy & None & 8 & Deceased \\
\hline Present & 66 & 56.78 & Biopsy & Surgery & 14 & Alive \\
\hline
\end{tabular}

SCCP, small-cell carcinoma of the prostate; PSA, prostate-specific antigen; TURP, transurethral resection of the prostate.

results of biochemical examinations were normal, except for the increased PSA level. A large proportion of cases diagnosed with pure SCCP usually have a PSA level within the normal range (11); however, the serum PSA level in the present case was significantly higher than normal $(56.78 \mathrm{ng} / \mathrm{ml}$; normal, $<4 \mathrm{ng} / \mathrm{ml}$ ).

The mean survival of patients with pure SCCP ranges between 4 and 12 months, with $<2 \%$ of the patients surviving beyond 12 months (1). The difficulty in the treatment of pure SCCP is due to its uncommon aggressive characteristics, similar to patients with SCCL. Pure SCCP is more common among older adults, with a mean age of 70 years at the time of diagnosis (7). However, the patient in this case was aged 66 years, which was younger compared with the mean reported age, and he survived beyond 12 months. The general rationale of SCCP treatment mainly includes radical surgery, chemotherapy, radiotherapy and endocrine therapy. Among all published cases, there is only one disease-free survival case that did not undergo radical surgery and was only treated with chemotherapy, without receiving other treatments, such as radiochemotherapy or endocrine therapy (2). Other researchers have suggested that surgery may not be the optimal choice, as patients with pure SCCP usually have distant metastasis at the time of the initial diagnosis (12). Furthermore, there remains the question of whether pure SCCP should be treated with only a combination of the chemotherapeutic agents that are applied in other cases of small-cell cancers. It has been reported that endocrine therapy or systemic chemotherapy may have some effect on the natural history of the disease (13).

To conclude, pure SCCP is rare, and the patient in the present case had a serum PSA level that was the highest reported to date. The patient has remained disease-free 14 months postoperatively, as determined at the last follow-up appointment during March, 2016. The findings of the present case raise the question of whether the PSA level is a trustworthy marker for the screening of the SCCP. Therefore, this case may be valuable for future studies.

\section{Acknowledgements}

Not applicable.

\section{Funding}

The present study was supported by the Science and Technology Development Fund Project of Shenzhen (grant nos. JCYJ20150403091443329 and JCYJ20170307111334308), the fund of the 'San-ming' Project of Medicine in Shenzhen (no. SZSM201612066), and the fund of Guangdong Key Medical Subject. 


\section{Availability of data and materials}

The datasets used or analysed during the current study are available from the corresponding author on reasonable request.

\section{Authors' contributions}

XMM approved the use of the patient for the case report. JH analysed patient data and was involved in drafting the manuscript as well as its critical revision for important intellectual content. TH performed follow-up appointments and collected patient data. LJ, YZ and WL performed data analysis. BW performed post-operative clinical examination of the patient. YL performed literature searches and collected patient data. YQL revised the manuscript critically for important intellectual content. LCN provided final approval of the manuscript version to be published. All authors read and approved the final manuscript.

\section{Ethics approval and consent to participate}

The present study was approved by the Ethics Committee of Peking University Shenzhen Hospital, and written informed consent was obtained from the patient.

\section{Consent for publication}

The patient provided informed consent for the use of the data in this study.

\section{Competing interests}

The authors declare that they have no competing interests.

\section{References}

1. Guo A, Wen S, Ma Y, Wei L and Liu A: Clinicopathological analysis on small cell carcinoma of the prostate in chinese patients. J Cancer 5: 797-803, 2014.
2. Horie K, Kameyama K, Mizutani K, Sugawara T, Seike K, Tsuchiya T, Yasuda M, Yokoi S, Nakano M, Deguchi T, et al: Small cell carcinoma of the prostate effectively treated for relatively long term: A case report. Hinyokika Kiyo 60: 517-521, 2014 (In Japanese).

3. Addeo A, Rinaldi C and Panades M: A case of small cell carcinoma of the prostate and review of the literature. Tumori 98: $76 \mathrm{e}-78 \mathrm{e}, 2012$

4. Hirai M, Konishi T, Saito K, Washino S, Kobayashi Y, Nokubi M, and Miyagawa T: Small cell carcinoma of the prostate: A case report of relative long-term survival. Nippon Hinyokika Gakkai Zasshi 106: 280-284, 2015 (In Japanese).

5. Kimura H, Uegaki M, Aoyama T, Kawai J, Hamano T and Hashimura T: Carboplatin plus irinotecan induced partial response in a patient with small cell carcinoma of the prostate; a case report. Hinyokika Kiyo 60: 39-43, 2014 (In Japanese).

6. Lee WY, Butt M, Campbell A and Greenstone M: Small cell carcinoma of the prostate and the syndrome of inappropriate antidiuretic hormone: A rare entity and presentation. Isr Med Assoc J 16: 458-460, 2014.

7. Dixit S, Coup A, Hunt C and Coombs L: Small cell cancer of the prostate. Urology 80: e58-e60, 2012.

8. Terada T: Primary small cell carcinoma of prostate without immunoreactive neuroendocrine proteins but with expressions of KIT and platelet-derived growth factor- $\alpha$. Int J Urol 22: 122-124, 2015.

9. Wang L, Davidson DD, Montironi R, Lopez-Beltran A, Zhang S, Williamson SR, MacLennan GT, Wang C, Wang M, Emerson RE, et al: Small cell carcinoma of the prostate: Molecular basis and clinical implications. Histol Histopathol 30: 413-424, 2015 (In Japanese).

10. Ishii G, Omono H, Kasai K, Hata K, Kimura T, Suzuki M and Egawa S: Docetaxel for small cell carcinoma of the prostate with a metastatic pelvic tumor: A case report. Hinyokika Kiyo 60: 641-644, 2014 (In Japanese).

11. Weiner AB, Patel SG, Richards KA, Szmulewitz RZ and Eggener SE: Population-based analysis of treatment modalities and survival for clinically localized small-cell carcinoma of the prostate. Prostate Cancer Prostatic Dis 17: 286-291, 2014.

12. Cohen A, Richards K A, PATEL S, et al: Metastatic small cell carcinoma of the prostate: Population-based analysis of patient characteristics and treatment paradigms. Urol Oncol 33: 70 e1-7, 2015.

13. Wang L, Williamson SR, Zhang S, Huang J, Montironi R, Davison DD, Wang M, Yao JL, Lopez-Beltran A, Osunkoya AO, et al: Increased androgen receptor gene copy number is associated with TMPRSS2-ERG rearrangement in prostatic small cell carcinoma. Mol Carcinog 54: 900-907, 2015. 